\title{
Téoros
}

Revue de recherche en tourisme

\section{Le Bureau international du tourisme social}

Quarante ans au service du droit aux vacances et au tourisme

\section{Charles-Étienne Bélanger}

Volume 22, numéro 3, automne 2003

L’accessibilité, une conquête inachevée

URI : https://id.erudit.org/iderudit/1071344ar

DOI : https://doi.org/10.7202/1071344ar

Aller au sommaire du numéro

Éditeur(s)

Université du Québec à Montréal

ISSN

0712-8657 (imprimé)

1923-2705 (numérique)

Découvrir la revue

Citer cet article

Bélanger, C.-É. (2003). Le Bureau international du tourisme social : quarante ans au service du droit aux vacances et au tourisme. Téoros, 22(3), 24-28.

https://doi.org/10.7202/1071344ar d'utilisation que vous pouvez consulter en ligne.

https://apropos.erudit.org/fr/usagers/politique-dutilisation/ 


\section{Le Bureau international du tourisme social}

\section{Quarante ans au service du droit aux vacances et au tourisme}

\section{Charles-Étienne Bélanger}

$\Delta$ lors que les voyages font partie intégrante des habitudes de vie de nos sociétés, nous oublions souvent qu'il existe encore une très large partie de la population pour qui le droit aux vacances et au tourisme demeure une utopie ou un rêve éloigné. C'est justement pourquoi la mission du Bureau international du tourisme social (BITS), qui consiste à promouvoir l'accès aux loisirs, aux vacances et au tourisme pour le plus grand nombre et à favoriser la mise en œuvre de cet objectif et des moyens qui en découlent auprès des acteurs qui partagent cette responsabilité, est toujours d'actualité. Pour le BITS, qui célèbre cette année ses quarante ans d'existence, l'ambition d'un tourisme pour tous, durable et solidaire, constitue le leitmotiv des actions à mener comme acteur à part entière dans le secteur du tourisme.

\section{Une action déterminante pour l'essor du tourisme social}

Pour bien saisir l'importance de l'action du BITS, il est utile de la situer dans le contexte qui a prévalu à l'essor du tourisme social. En effet, au lendemain de la Seconde Guerre mondiale, pour un nombre croissant d'individus le besoin de partir en vacances a pris une certaine ampleur, donnant lieu à la création d'organismes - associations sans but lucratif, syndicats, coopératives, mouvements de jeunesse - qui, soucieux de proposer des options de vacances à la population, se sont par la suite regroupés tant à l'échelle nationale qu'internationale. Avec l'a- vènement des premiers congés payés en 1936, acquis sous la pression des mouvement ouvriers, et leur reconnaissance dans la Déclaration universelle des Droits de l'Homme en 1948, le développement des loisirs et du tourisme connâ̂tra une croissance rapide et donnera lieu, dans certains pays, à la naissance de véritables politiques sociales du tourisme. Ces politiques sociales du tourisme

se traduiront par des interventions dans deux grands champs d'activité, connus sous les vocables de l'aide à la pierre et l'aide à la personne. Dans le premier cas, il s'agit de l'aide au développement (construction, rénovation et modernisation) d'équipements et infrastructures alors que dans le second, les mesures visent les personnes (faciliter les départs en vacances). Dans un cas comme dans l'autre, des formules et des expériences novatrices verront le jour et permettront à des millions de personnes de mettre en pratique le droit aux vacances. On parlera alors d'une démocratisation des voyages et du tourisme qui connấtra des taux de croissance particulièrement élevés (Bélanger, 1999).

C'est dans ce contexte que le BITS sera créé en 1963 par un certain nombre d'organismes dans le but de se doter d'un forum permanent qui leur permette de débattre à l'échelle internationale des questions liées au tourisme social. Parmi les grands thèmes qui seront abordés au sein du BITS, il faut mentionner le financement des vacances, le tourisme des jeunes (Manifeste du tourisme des jeunes, 1967), le tourisme et la culture, le tourisme familial, le tourisme et l'environnement (Acte de Copenhague, 1982), l'étalement des vacances (Propositions d'action pour la Communauté européenne, 1983), le tourisme en milieu rural, le tourisme comme facteur de développement et d'intégration, les aides au départ en vacances dans l'Union européenne et plusieurs autres thèmes qui marqueront les congrès biennaux du BITS et donneront lieu à de nombreuses publications.

L'influence du BITS se fera aussi sentir au sein d'organisations internationales comme l'Organisation mondiale du tourisme (OMT) où la contribution du BITS sera particulièrement significative dans le processus qui aboutira à l'adoption, en 1980, de la Déclaration de Manille sur le tourisme mondial à laquelle ont souscrit 107 États et 91 observateurs.

La Déclaration de Manille a synthétisé les idées fondamentales sur la nature et les bénéfices du tourisme, idées qui sont aujourd'hui généralement partagées par la communauté internationale. Non seulement la Déclaration proclame le droit des personnes à prendre des vacances mais elle exprime aussi la conviction - inscrite dans les Statuts de l'OMT - que le tourisme peut jouer un rôle indispensable dans la promotion de la croissance économique et sociale des pays en développement ${ }^{1}$.

Aujourd'hui, les questions liées au droit au tourisme pour tous sont inscrites au Code mondial d'éthique du tourisme, adopté par l'OMT en 1999.

Si l'action du BITS a été déterminante pour l'essor du tourisme social et qu'elle 
a marqué les grands débats internationaux sur cet enjeu, elle a aussi été importante dans l'actualisation du concept même du tourisme social qui a dû se renouveler au cours des années.

\section{La mise à jour du concept de tourisme social}

Au-delà de ses statuts dans lesquels le tourisme social est défini comme «l'ensemble des rapports et des phénomènes résultant de la participation au tourisme et en particulier de la participation des couches sociales à revenus modestes [et que] cette participation est rendue possible, ou facilitée, par des mesures d'un caractère social bien défini ${ }^{2}{ }^{2}$, le BITS a rapidement vu la nécessité d'adopter un premier texte de référence pour mieux préciser son action et celle des organismes responsables du tourisme social.

C'est ainsi qu'en 1972, à l'occasion de son Assemblée générale à Vienne, le BITS adoptera la Charte de Vienne qui présente le tourisme comme un «fait social fondamental de notre temps » et énumère des principes d'action selon lesquels « le tourisme doit traduire une volonté d'épanouissement, de maturation humaine et qu'il doit rester une affirmation de la liberté de l'individu ». On y mentionne aussi la nécessité « de poursuivre l'effort permanent d'éducation et d'information objective des masses intéressées, seul susceptible de conserver au Tourisme la vocation humaniste qui lui appartient ». Enfin, on y affirme «qu'aucune politique sociale ne saurait se concevoir sans comprendre une politique sociale du tourisme qui doit retenir l'attention de tous les gouvernements $»^{3}$.

Ce texte, pour reprendre les propos du professeur Louis Jolin (2003), reflète « une époque où plusieurs promoteurs $d u$ tourisme social revendiquaient pour ce secteur une fonction socioéducative, voire même sociopolitique. L'animation, inspirée de celle qui a cours dans les mouvements d'éducation populaire, avait une forte dimension militante ».

Au cours des années 1980, l'environnement mondial connaîtra de profonds bouleversements qui affecteront le secteur du tourisme social ${ }^{4}$. En effet, cette période sera caractérisée par le triomphe de l'idéologie néolibérale et de la libre concurrence, une réduction du rôle des États, la crise économique et l'impératif d'hyper compétitivité ; on assistera alors à une diminution des budgets de nombreux États, à une remise en cause de leurs politiques sociales et au développement de sociétés à deux vitesses avec la montée du chômage, l'exclusion sociale et les problèmes d'intégration des migrants.

Les effets directs sur le secteur du tourisme social ne se feront pas attendre :

- la quasi-disparition des aides publiques à l'investissement en raison des restrictions budgétaires ;

- le nouvel impératif pour les associations de se transformer en véritables entreprises à vocation sociale ;

- la nécessité pour les associations de se tourner vers de nouvelles clientèles solvables.

Ces transformations amèneront donc les acteurs du tourisme social à se repositionner et le BITS à mener une réflexion pour actualiser le concept de tourisme social qui tiendra compte du nouveau contexte mondial marqué par l'effondrement des économies socialistes et l'émergence d'un tourisme « pour tous » dans certains pays d'Amérique latine, d'Afrique et d'Asie.

Cette réflexion aboutira en 1996 au texte de la Déclaration de Montréal «Pour une vision humaniste et sociale du tourisme » qui se distingue essentiellement de la Charte de Vienne par le fait qu'elle enjoint les acteurs à « une gestion exemplaire », qu'elle préconise un développement durable et soutenu, «un tourisme maîtrisé et respectueux des sites et des populations » et qu'elle redéfinit les critères d'identification du tourisme social en affirmant que «ce ne sont pas les statuts juridiques ou les procédures utilisées qui légitiment les opérateurs touristiques mais l'action qu'ils conduisent au service d'un objectif clairement affirmé et poursuivi »(BITS, 1996).

Comme l'observe Jolin (2003 : 8), il est intéressant de noter qu'en plus de l'accessi- bilité au tourisme, la Déclaration de Montréal introduit une dimension de solidarité entre les touristes et les populations locales et confirme que « le tourisme social est tout le contraire d'un tourisme de masse envahisseur et spoliateur des ressources ».

\section{Les nouveaux enjeux}

C'est sur la base de ce texte de référence que les enjeux actuels du BITS peuvent être présentés. Ils se définissent sur trois plans : le tourisme pour tous, le tourisme solidaire et le défi des moyens".

\section{Le tourisme pour tous}

Le tourisme pour tous concerne bien l'ambition de faire accéder de plus en plus de monde à la réalité du tourisme et des loisirs puisque, encore aujourd'hui, même dans un pays comme la France qui est la première destination touristique mondiale et qui possède pourtant une politique sociale du tourisme bien développée, près de $40 \%$ des Français ne partent toujours pas en vacances. Cette proportion de non-départs, on le comprend aisément, est encore beaucoup plus élevée dans les pays émergents.

Le tourisme pour tous doit donc continuer à être la priorité du BITS et des différents acteurs nationaux, à savoir les États, les acteurs sociaux et les opérateurs. Or, et c'est justement là un des problèmes, dans plusieurs pays on observe une absence totale de politiques sociales en matière de tourisme, que les acteurs sociaux, à eux seuls, ne peuvent combler. Il en va du rôle de l'État de favoriser et d'appuyer le départ en vacances des diverses couches de la population et de trouver des stratégies adaptées aux diverses clientèles.

Les jeunes, les familles, les personnes âgées et les personnes à capacité physique restreinte constituent les principales clientèles concernées par une politique sociale du tourisme qui a aussi des répercussions économiques non négligeables. En ce qui concerne le tourisme des jeunes, par exemple, il est intéressant de noter que dans un nombre croissant de pays on développe des stratégies destinées à mieux informer cette clientèle des diverses options qui lui sont offertes et à 
se positionner sur les marchés internationaux comme une «destination jeune ».

Il en est de même pour le tourisme familial. Le développement d'une offre en matière d'hébergement, d'animation et d'activités, adaptée aux besoins des divers types de familles et combinée à des mesures d'aide au départ en vacances, permet à des milliers de personnes de concrétiser leur «droit aux vacances ». D'autre types de besoins appellent aussi à des réponses pour certaines couches de la population. C'est le cas des populations immigrées pour qui le temps des vacances évolue au fil de leur intégration à la société d'accueil et dont il importe de considérer certaines spécificités ${ }^{6}$.

La prise en compte du tourisme pour tous dans la politique touristique et une plus grande coopération entre les secteurs public et privé sont, et seront pour l'avenir, une priorité dans les actions du BITS. Parmi les premiers pays récepteurs de touristes au monde, plusieurs disposent d'une politique sociale du tourisme, au niveau central et/ou régional comme c'est le cas de la France, de l'Espagne et du Mexique. Ces données ne constituent certes pas un hasard et démontrent que le tourisme social peut servir de moteur pour le développement d'un tourisme national et être complémentaire à une stratégie axée sur le développement des marchés internationaux. Il est dommage de constater que dans certains pays, principalement émergents, la mise en pratique d'une politique d'accès aux vacances ne se réalise qu'en période de crise, lorsque les flux touristiques étrangers diminuent en raison de conflits ou de crises qui surgissent de façon périodique.

\section{Le tourisme solidaire}

Le tourisme solidaire dont la «finalité est d'amener le touriste à une forme de solidarité concrète avec les populations visitées [et qui] peut prendre plusieurs aspects comme, par exemple, le soutien à un projet de développement ou la participation à un fonds d'entraide » (UNAT ${ }^{7}$, 2002) constitue un nouvel enjeu pour le BITS et un chantier dans lequel l'organisation entend assumer un certain leaders- hip. Mais, pourquoi un organisme voué à la promotion d'un tourisme pour le plus grand nombre s'intéresse-t-il à une forme de tourisme qui, par sa nature, se pratique en petits groupes et est souvent loin d'être accessible en termes de coûts ?

Le premier élément de réponse s'explique par le fait que le tourisme social tel que défini dans la Déclaration de Montréal s'appuie sur les valeurs intrinsèques du tourisme durable qui est à la base même du tourisme solidaire. Le tourisme durable repose sur les trois grands principes suivants : «un tourisme supportable à long terme sur le plan écologique, équitable au plan éthique et social et viable sur le plan économique » (Caire et Rouillet-Caire 2003).

La deuxième explication repose sur le fait que l'accès aux sites et aux ressources touristiques et aux bénéfices du tourisme doit être une réalité non seulement pour les visiteurs et les étrangers, mais aussi pour les populations d'accueil. Dans ce domaine, on le sait fort bien, beaucoup reste encore à faire puisque les retombées du tourisme ne sont pas toujours évidentes quand on se rend compte de l'état dans lequel vivent certaines populations à proximité de zones touristiques.

Enfin, il faut reconnaître que les outils qui ont été développés pour le tourisme pour tous peuvent être utilisés pour un tourisme spécialisé qui intègre des propositions de tourisme solidaire. En ce sens, les réseaux associatifs et le secteur des coopératives peuvent jouer un certain rôle dans la promotion et la commercialisation d'une offre particulière par l'intermédiaire de leurs réseaux de membres.

Aujourd'hui, même s'il est vrai que les associations de tourisme social au sein du BITS se préoccupent de solidarité avec les communautés d'accueil, le BITS est conscient que nombre d'associations et d'ONG (organisations non gouvernementales) ont vu le jour ces dernières années en dehors de son réseau, d'où l'intérêt à encourager un rapprochement entre le milieu associatif et celui des ONG afin de créer un lieu permanent de concertation et de discus- sions. Les récentes initiatives du BITS et de certains de ses membres vont d'ailleurs dans ce sens :

- le travail de l'UNAT en France : création d'un comité sur le tourisme solidaire, publication de la brochure $D$ 'autres voyages - du tourisme à l'échange, co-organisation avec l'Association malienne pour la promotion du tourisme social (AMPTS) d'un séminaire sur les concepts de tourisme social et de tourisme solidaire à Bamako et co-organisation du premier Forum international sur le tourisme solidaire (FITS) à Marseille en septembre 2003 ;

- la Charte du Touriste Responsable et les différentes publications et actions que certaines associations italiennes, adhérentes au BITS (Legacoop, CTG, CTS), ont mises en place avec l'Association italienne de tourisme responsable (AITR);

- le Séminaire sur le tourisme solidaire organisé en juin 2003 à Montréal par le Secrétariat du BITS pour les Amériques ;

- la participation du BITS au projet interdisciplinaire pour l'élimination de la pauvreté coordonné par l'UNESCO et s'intitulant Stratégie pour un développement durable du tourisme au Sahara.

Les défis liés au développement du tourisme solidaire sont nombreux et concernent notamment la définition de critères d'identification, la mise en réseau, la diffusion des bonnes pratiques, la commercialisation des diverses initiatives ainsi que la diversification de la clientèle qui constituent autant d'aspects qui peuvent contribuer «au passage du développement du tourisme au tourisme de développement » (Tonini, 2003)

\section{Le défi des moyens}

Ce troisième défi n'est pas le moindre puisqu'il nécessite la création de réseaux et de mises en marché de tout ce qui s'inscrit dans les ambitions d'un tourisme social et d'un tourisme solidaire.

Comme plusieurs des associations et des autres organismes sont souvent nés d'initiatives locales ou nationales, ils doivent s'intégrer à un véritable réseau à l'échelle internationale : 


\begin{abstract}
Il nous faut relever le défi d'une reconstruction ou d'une construction d'un partenariat dynamique avec les organisations internationales, les pouvoirs publics (États, collectivités régionales ou locales), avec les partenaires sociaux, en premier lieu les mouvements syndicaux et le monde de l'économie sociale ou du tiers secteur: les mouvements coopératifs, les community development corporations et tant d'autres... (Mignon, 2002).
\end{abstract}

Enfin, une des conditions importantes pour relever le défi consiste à situer les actions au cœur même de l'industrie touristique en apportant des idées et en proposant des actions complémentaires à ce que font les autres secteurs. Il est plus facile de se faire reconnaître en apportant un discours constructif et en prenant part aux manifestations importantes organisées dans le domaine du tourisme plutôt qu'en s'opposant à ce que font les autres secteurs professionnels du tourisme, ce qui n'exclut pas qu'il faille dénoncer les abus qui entraînent une dégradation des conditions favorables à un développement durable et équitable du tourisme.

\section{Le BITS aujourd'hui : la force d'un réseau}

Pour mener à bien sa mission, le BITS compte sur la force de son réseau qui regroupe près de 140 organismes situés dans 35 pays différents, principalement en Europe et dans les Amériques. Une des richesses du BITS repose sur la diversité de ses membres parmi lesquels on trouve surtout, sur le plan juridique, des associations à but non lucratif, des coopératives, des établissements publics et, sur le plan opérationnel, des réseaux de centres de vacances et d'auberges de jeunesse, des agences et opérateurs de voyages, des institutions d'enseignement, des organisation syndicales, des ONG de coopération et des organismes officiels de tourisme qui exercent tous une activité utile au tourisme social.

Les actions du BITS se concrétisent principalement par la diffusion d'information, par l'organisation d'événements dont la principale manifestation est le congrès mondial qui se déroule tous les deux ans, par la participation à des recherches, par des projets de coopération et des missions d'expertise ainsi que par des activités de lobbying.

Une des rôles du BITS consiste à diffuser de l'information sur toute l'actualité dans le domaine du tourisme social et à favoriser les contacts et les échanges entre les membres et les partenaires. Pour ce faire, le BITS dispose d'un ensemble d'outils de communication comme le bulletin électronique mensuel Info BITS, le magazine Le tourisme social dans le monde qui publie des dossiers de fond sur des sujets aussi divers que le tourisme coopératif, l'accès au tourisme des personnes handicapées, le tourisme et l'économie sociale, etc. La promotion du droit aux vacances pour tous se fait aussi d'une façon permanente sur le site Internet du BITS [http://www. bits-int.org] où l'on trouve une quantité de renseignements sur les activités, les projets, les initiatives de l'organisation ainsi que des liens électroniques avec les sites respectifs des membres. Enfin, il y a le Who is Who, le répertoire de l'organisation, qui présente une description des activités de chaque organisme membre.

En ce qui concerne l'organisation d'événements, le BITS est aussi très actif en dehors des congrès biennaux. Au cours des dernières années, à titre d'exemples, le Secrétariat du BITS pour les Amériques a organisé trois séminaires sur la thématique du tourisme des jeunes à Montréal (1995), à Mexico (1999) et à Toronto (2001), un séminaire sur le tourisme pour tous à Morelia au Mexique, dans le cadre d'une réunion du groupe de travail en tourisme de l'APEC (Asia Pacific Economic Cooperation), et un Colloque sur le chèque-vacances au Québec en 2000.

La section européenne du BITS a aussi organisé des manifestations importantes au cours des dernières années, dont un séminaire sur les aides au départ en vacances dans l'Union européenne à Strasbourg (2000) et, en collaboration avec Tourisme Flandres, une Conférence européenne ministérielle sur le « touris- me pour tous » à Bruges en 2001. Enfin, en 2002, le BITS co-organisait, sur l'initiative de l'IAST (International Association for Social Tourism), un premier forum sur le tourisme dans les pays d'Europe centrale et orientale (PECO) à Zakopane en Pologne.

Le réseau du BITS est aussi utile pour développer des contacts et faciliter les échanges entre les organismes qui souhaitent créer des occasions d'affaires. C'est pourquoi le BITS, avec ses partenaires que sont l'IAST et la TICA (Tourism International Cooperative Association), organise depuis quelques années des workshops à caractère commercial, lesquels constituent une excellente vitrine pour la promotion de produits et de services touristiques.

\section{Conclusion}

En conclusion, nous pouvons affirmer que le secteur du tourisme social, porteur d'une vision humaniste du tourisme, représente des millions de personnes et est sans doute le créneau le plus prometteur des dernières années. Sur l'Europe, de nouvelles possibilités s'ouvriront avec l'élargissement de l'Union européenne. En Amérique latine, le potentiel de développement qui a commencé à se manifester ces dernières années se poursuivra sans aucun doute. Quant à l'Afrique et à l'Asie, même si le concept de tourisme social y est peu connu, de nombreuses pistes, notamment grâce aux mouvements coopératifs et au community-based tourism, indiquent que nombre d'organismes partagent les mêmes besoins que ceux du secteur du tourisme social. Dans ce contexte, il n'est pas trop optimiste de penser que le BITS a encore devant lui quarante autres années pour poursuivre sa mission de promouvoir un tourisme pour tous, durable et solidaire.

Charles-Étienne Bélanger est directeur du BITS au siège social à Bruxelles.

\section{Notes}

1 Luigi Cabrini, représentant régional de l'OMT pour l'Europe, Discours prononcé à l'occasion du $40^{\mathrm{e}}$ anniversaire du BITS le 13 juin 2003. 
2 BITS, Texte des statuts adoptés en 1963 et révisés pour la dernière fois lors de l'Assemblée générale de Mexico, 15 mai 2002.

3 BITS, Charte de Vienne adoptée au Congrès du BITS de 1972.

4 Voir à ce sujet l'analyse de Froidure (1997).

5 Voir à ce sujet Mignon (2002).

6 Voir le numéro spécial de la revue Hommes et migration, « Le temps des vacances ».

7 Union nationale des associations de tourisme et de plein air.

\section{Bibliographie}

« Le temps des vacances », Hommes et migration, numéro spécial, n 1243, mai-juin 2003.

Association italienne de tourisme responsable (AITR) (1997), Charte du tourisme responsable.
Bélanger, Charles-Etienne (1999), « Le tourisme social - Bilan, enjeux et perspectives », Téoros, vol. 18, n 3, p. 53.

BITS (2002), Actes du Congrès de Mexico, CD-Rom.

BITS (1972), Charte de Vienne, adoptée au Congrès du BITS.

BITS (1996), Déclaration de Montréal, Pour une vision humaniste et sociale du tourisme.

BITS (1983), L'étalement des vacances, Propositions d'action pour la Communauté européenne, vol. I et vol. II, Bruxelles.

BITS (1967), Manifeste du tourisme des jeunes, adopté lors du congrès spécial du BITS tenu à Fiuggi, Italie.

BITS, Statuts, adoptés en 1963 et modifiés à quelques reprises, la dernière modification remontant à mai 2002.

BITS (1982), Tourisme et Environnement, Acte de Copenhague.

Caire, Gilles, et Monique Rouillet-Caire (2003), Tourisme du Nord et développement durable du Sud : la contribution de l'" alter-tourisme », Conférence prononcée lors du Forum internatio- nal sur le tourisme solidaire (FITS), Marseille, septembre.

Froidure, Jean (1997), Du tourisme social au tourisme associatif, Paris, l'Harmattan.

Jolin, Louis (2003), « Le tourisme social, un concept riche de ses évolutions ", Le tourisme social dans le monde, Édition spéciale $40^{\mathrm{e}}$ anniversaire, no 141, p. 7.

Mignon, Jean-Marc (2002), Tendances et défis du tourisme social, Actes du Congrès du BITS à Mexico.

OMT (1999), Code mondial d'éthique du tourisme, Adopté lors de l'Assemblée générale de l'OMT à Santiago du Chili.

OMT (1980), Déclaration de Manille sur le tourisme mondial, Adoptée par la Conférence mondiale sur le tourisme.

Tonini, Norberto (2003), L'apport du BITS pour le développement du tourisme solidaire et équitable, Conférence prononcée lors du Forum international sur le tourisme solidaire, Marseille.

UNAT (2002), D'autres voyages du tourisme à l'échange, p. 49.

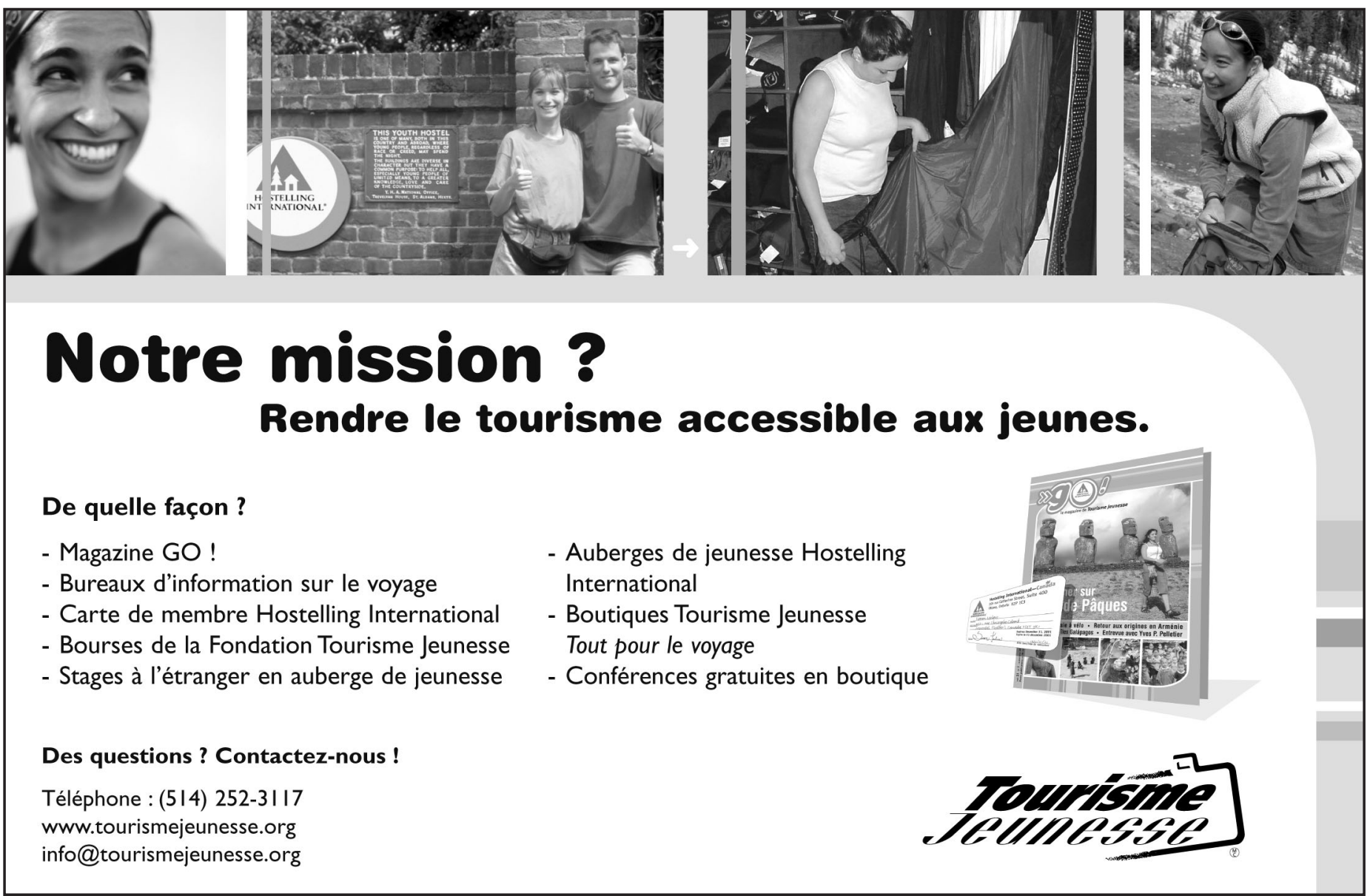

